\title{
EXTENSION OF FUNCTORS TO FIBREWISE POINTED SPACES
}

\author{
Petar Pavešić
}

\begin{abstract}
We describe a new general method for the fibrewise extension of a given endofunctor on the category of pointed topological spaces to the category of fibrewise pointed spaces. We derive some properties of the construction and show how it can be profitably used to build the WhiteheadGanea framework for the fibrewise Lusternik-Schnirelmann category and the topological complexity.
\end{abstract}

\section{Introduction}

This paper has two objectives. The first is to introduce a new method for the fibrewise extension of a functor defined on the category of pointed topological spaces to a functor on the category of fibrewise pointed spaces. More precisely, given a fibrewise space $p: X \rightarrow B$ with a prescribed section $s: B \rightarrow X$ (so that for every $b \in B$ we consider $s(b)$ as the base-point of $X_{b}=p^{-1}(b)$ ), and a continuous endofunctor $\Phi: \operatorname{Top}_{*} \rightarrow \operatorname{Top}_{*}$ on the category of pointed topological spaces we construct a fibrewise pointed space $X[\Phi] \rightarrow B$ whose fibres are precisely the spaces $\Phi\left(X_{b}, s(b)\right)$. The construction is very general, it has many pleasant properties and lot of applications. In the second part we present one of the applications, namely the construction of the Whitehead-Ganea framework for the fibrewise Lusternik-Schnirelmann category, and in particular for the topological

2010 Mathematics Subject Classification. 55R70, 55M30.

Key words and phrases. Fibrewise pointed space, continuous functor, LusternikSchnirelmann category, topological complexity.

The author was supported by the Slovenian Research Agency grant P1-02920101. 
complexity. The precise definitions and some historical perspective are given in the respective sections.

\section{Extension of functors}

We will use freely the standard terminology and notation of the theory of fibrewise spaces as in [9], [11] and [12]. In particular, given a topological space $B$ a fibrewise space over the base $B$ consists of a total space $X$ together with a continuous projection map $p: X \rightarrow B$. For each $b \in B$ the preimage $X_{b}:=$ $p^{-1}(b)$ is called the fibre over $b$. A morphism between fibrewise spaces $X$ and $Y$ over $B$ is a map $f: X \rightarrow Y$ that commute with the projections.

A fibrewise space $X$ is pointed if there exists a continuous section map $s: B \rightarrow X$, such that $p \circ s=\mathrm{I}_{B}$. A morphism between fibrewise pointed spaces $X$ and $Y$ is a map $f: X \rightarrow Y$ that commute with the projections and sections.

Intuitively, a fibrewise space $X$ is a continuous family of spaces $X_{b}$ (parametrized by the elements of $B$ ), while a fibrewise pointed space is a continuous $B$-parametrized family of pointed spaces $\left(X_{b}, s(b)\right)$.

When dealing with fibre spaces (vector bundles, fibre bundles, fibrations, ... ) it is often necessary to construct a fibrewise extension of an operation which is defined on a certain class of spaces to an operation on the fibre spaces whose fibres are elements of that class. Classical examples are the constructions of tensor bundles and the bundles of differential forms as in [6, Section 5.6], which rely on the local triviality structure of vector bundles. For general fibrewise spacea James describes a very simple construction that extends a continuous endofunctor on the category of topological spaces to the fibres of any fibrewise space. Since it inspired our construction we provide a few details. Let $p: X \rightarrow B$ be a fibrewise space and let $\Phi:$ Top $\rightarrow$ Top be a continuous functor (to be defined later). Then, following $[9$, p. 76$]$ one defines $\Phi_{B}(X)$ to be the set $\coprod_{b \in B} \Phi\left(X_{b}\right)$ with the initial topology determined by the function

$$
\coprod_{b \in B} \Phi\left(X_{b}\right) \rightarrow \coprod_{b \in B} \Phi(X) \equiv B \times \Phi(X)
$$

whose restriction on each $\Phi\left(X_{b}\right)$ is the map $\Phi\left(X_{b} \hookrightarrow X\right)$. Unfortunately, when $\Phi$ is a functor on the category of pointed spaces then the above construction does not work as it does not take into account the effect of the choice of the basepoint on the values of of the functor. This is why the subsequent developments of the fibrewise homotopy theory prefer to work with explicit constructions. For example Crabb amd James [2] describe explicitly fibrewise wedges, smashproducts and other pointed constructions. This is further generalized by May and Sigurdsson [11] who prove that every limit or colimit construction on the level of pointed spaces can be naturally extended to fibrewise pointed spaces. While this approach is sufficient for most applications it still lacks generality and 
complicates many arguments and proofs. On the other side Iwase and Sakai [7] consider an arbitrary continuous functor $\Phi$ but require that $X$ is locally trivial as a fibrewise pointed space. Apart from being relatively complicated, the main drawback of their construction is that many fibrewise pointed spaces that appear in practice are not locally trivial. Let us illustrate this important point with an example.

EXAMPLE 2.1. The fibrewise product of fibrewise pointed spaces

$$
B \stackrel{s}{\longrightarrow} X \stackrel{p}{\longrightarrow} B \quad \text { and } \quad B \stackrel{r}{\longrightarrow} Y \stackrel{q}{\longrightarrow} B
$$

is given by the space $X \times_{B} Y:=\{(x, y) \in X \times Y \mid p(x)=q(y)\}$ with obvious projection and section (cf. [2]). Moreover the fibrewise wedge is the following subspace of $X \times{ }_{B} Y$ :

$$
X \vee_{B} Y:=\{(x, y) \in X \times Y \mid x=s p(x) \text { or } y=r q(y)\}
$$

(intuitively, $X \vee_{B} Y$ is obtained by gluing together $X$ and $Y$ along the respective sections).

Let $X:=[0,1] \times[0,1]$ be the fibrewise pointed space with respect to the projection to the first component $\operatorname{pr}_{1}: X \rightarrow[0,1]$ and with the diagonal section $\Delta:[0,1] \rightarrow X$. Then $X$ is trivial as a fibrewise pointed space but is not even locally trivial as a fibrewise pointed space. This is best seen by observing that the fibres of $X \vee_{[0,1]} X$ over the points 0 and $1 / 2$ are not homeomorphic as it should be the case for a wedge of locally trivial spaces. The same problems arises with other constructions that are used in the fibrewise Lusternik-Schnirelmann category and topological complexity, which explains why the local triviality assumption should be avoided.

In order to describe our construction we need only a mild topological restriction and require the fibrewise pointed space $X$ to be a Tikhonov space (i.e. completely regular and Hausdorff). It is conceivable that this assumption may be somehow avoided but we have not be able to do so. It is well-known that every Tikhonov space can be embedded into a topological product of copies of the real line indexed over some basis of the topology of $X$. Let $E$ denote such a product and let $i: X \hookrightarrow E$ be the corresponding embedding. Moreover, as $E$ is a linear space we denote by + its continuous addition operation and by $0 \in E$ its origin.

Let $B \stackrel{s}{\longrightarrow} X \stackrel{p}{\longrightarrow} B$ be a fibrewise pointed space over $B$ and assume that $X$ is a Tikhonov space so that we may choose an embedding $i: X \hookrightarrow E$ and consider the family of maps

$$
i_{b}:\left(X_{b}, s(b)\right) \rightarrow(E, 0), \quad i_{b}(x):=i(x)-i(s(b)), \quad b \in B .
$$


For any functor $\Phi:$ Top $_{*} \rightarrow \operatorname{Top}_{*}$ we define the space $X[\Phi]$ as the set

$$
\coprod_{b \in B} \Phi\left(X_{b}, s(b)\right)
$$

endowed with the initial topology determined by the function

$$
\coprod_{b \in B} \Phi\left(i_{b}\right): \coprod_{b \in B} \Phi\left(X_{b}, s(b)\right) \rightarrow \coprod_{b \in B} \Phi(E, 0) \equiv B \times \Phi(E, 0) .
$$

The projection $p_{\Phi}: X[\Phi] \rightarrow B$ is given by mapping $\Phi\left(X_{b}, s(b)\right)$ to $b$ and it is continuous because $p_{\Phi}=\operatorname{pr}_{B} \circ \coprod \Phi\left(i_{b}\right)$, a composition of two continuous maps. The section $s_{\Phi}: B \rightarrow X$ sends $b \in B$ to the base point of $\Phi\left(X_{b}, s(b)\right)$, and its continuity follows from the universal property of the initial topologies. We conclude that

$$
B \stackrel{s_{\Phi}}{\longrightarrow} X[\Phi] \stackrel{p_{\Phi}}{\longrightarrow} B
$$

is a fibrewise pointed space over $B$.

By definition, the fibre of $X[\Phi]$ over $b \in B$ has the initial topology with respect to the map $\Phi\left(i_{b}\right)$, and that topology may be in general stronger than the topology of $\Phi\left(X_{b}, s(b)\right)$. We will say that a functor $\Phi:$ Top $_{*} \rightarrow$ Top $_{*}$ preserves real subspaces if for every $A$ embedded as a subspace $i: A \hookrightarrow E$ of some product of real lines $E$, and for every $a \in A$ the space $\Phi(A, a)$ has the initial topology with respect to the map $\Phi(i)$. When the functor $\Phi$ preserves injective maps than the above condition simply means that $\Phi(A, a)$ is a subspace of $\Phi(E, a)$.

One can easily verify that standard endofunctors on $\mathrm{Top}_{*}$, like products, (fat) wedges, based path spaces, based loop spaces and many others preserve real subspaces. There are also functors that do not preserve subspaces, e.g. the one-point compactification (observe that the one-point compactification of the rationals is not a subspace of the one-point compactification of the reals). In some cases, in particular when the description of $\Phi$ involves some identifications, it is necessary to assume that $A$ is a closed subset of $E$. Then it is sufficient to assume that $\Phi$ preserves closed real subspaces and that condition holds for functors like smash-products and joins.

Proposition 2.2. Assume that $\Phi:$ Top $_{*} \rightarrow$ Top $_{*}$ preserves (closed) real subspaces and that $X$ is a fibrewise pointed space over $B$ that admits a (closed) embedding into some product of real lines. Then $X[\Phi]$ is a fibrewise pointed space over $B$ whose fibres are precisely the values of $\Phi$ on the respective fibres of $X$.

A fundamental problem is to understand how the topology of $X[\Phi]$ depends on the choice of the embedding $i: X \hookrightarrow E$. It is clear that in order to preserve various topological properties the functor $\Phi$ must be in some sense continuous. A functor $\Phi: \operatorname{Top}_{*} \rightarrow \operatorname{Top}_{*}$ is said to be topologically continuous if it preserves continuous families of pointed maps. In detail, we require that for every space $A$, every pair $(X, x),(Y, y) \in \operatorname{Top}_{*}$ and every map $f:(A \times X, A \times\{x\}) \rightarrow(Y, y)$ 
the induced map $\widehat{f}: A \times \Phi(X, x) \rightarrow \Phi(Y, y)$, given by $\widehat{f}(a, u):=\Phi(f(a,-))(u)$ is also continuous. The attribute 'topologically' is used as a disambiguation, to avoid potential confusion with another common notion of continuity in category theory related to functors that preserve categorical limits. Again it is not difficult to check that the usual endofunctors on $\mathrm{Top}_{*}$ like products, wedges, path and loop spaces, smash-products and joins, are topologically continuous in the above sense.

THEOREM 2.3. Let $B \stackrel{s}{\longrightarrow} X \stackrel{p}{\longrightarrow} B$ be a fibrewise pointed space such that $X$ admits a closed embedding $i: X \hookrightarrow E$ into a countable product of real lines, and let $\Phi$ be an endofunctor on $\mathrm{Top}_{*}$ that is topologically continuous and preserves closed real subspaces. Then the topology of $X[\Phi]$ does not depend on the choice of the embedding $i$.

Proof. We first consider a special case. If $j:(E, 0) \hookrightarrow\left(E^{\prime}, 0\right)$ is a closed embedding of products of real lines, then the assumption that $\Phi$ preserves closed real subspaces implies that the topology on $X[\Phi]$ determined by the embedding $j \circ i: E^{\prime}$ coincides with the topology determined by $i$.

Toward the general case, let $i^{\prime}: X \rightarrow E^{\prime}$ be another closed embedding of $X$ into a countable product of real lines $E^{\prime}$. The products $E$ and $E^{\prime}$ are metrizable and therefore normal (which is not the case for uncountable products, cf. [13, Theorem 3]), and are at the same time absolute extensors for normal spaces, so there exist maps $f: E \rightarrow E^{\prime}$ and $g: E^{\prime} \rightarrow E$ such that the following diagram commutes

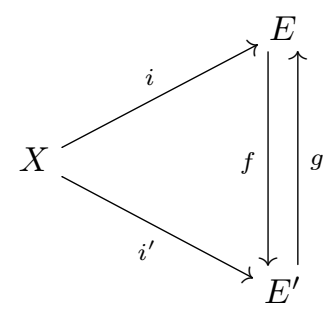

Furthermore, the map $h: E \times E^{\prime} \rightarrow E \times E^{\prime}, h(x, y):=(x-g(y+f(x)), y+f(x))$ is a homeomorphism (with inverse $h^{-1}(x, y)=(x+g(y), y-f(x+g(y))$, cf. [3]) that makes commutative the following diagram

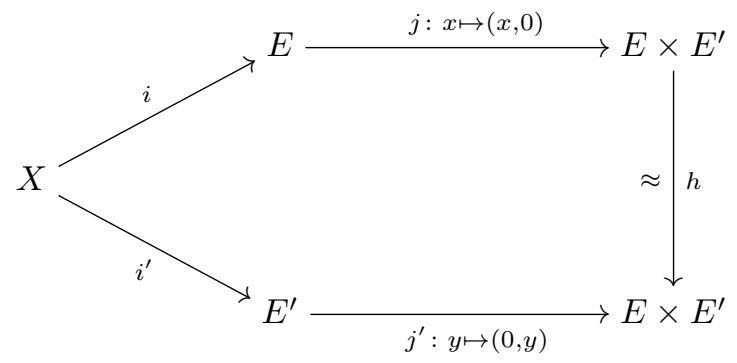


and this implies that the embeddings $j i, j^{\prime} i^{\prime}: X \rightarrow E \times E^{\prime}$ are ambient-homeomorphic.

Next we define a continuous family of maps $h_{b}:\left(E \times E^{\prime}, 0\right) \rightarrow\left(E \times E^{\prime}, 0\right)$ for $b \in B$ given by

$$
h_{b}(u):=h(u+j i s(b))-j^{\prime} i^{\prime} s(b)
$$

and check that $h_{b} \circ j \circ i_{b}=j^{\prime} \circ i_{b}^{\prime}$. By the continuity of $\Phi$ we get the following diagram

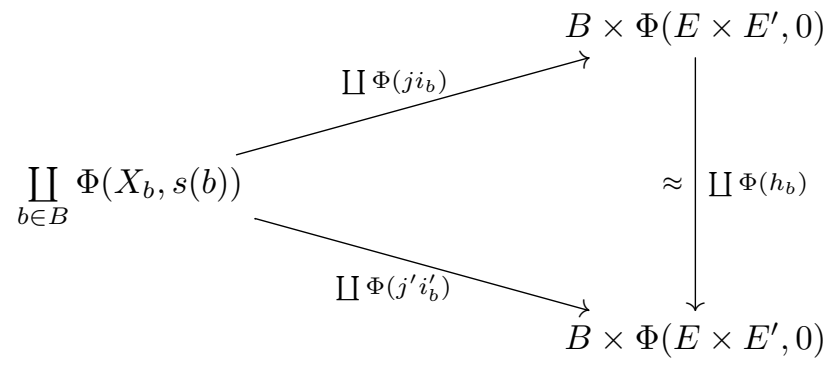

We conclude that the embeddings $j i$ and $j^{\prime} i^{\prime}$ induce the same topology on $X[\Phi]$, which in view of our initial remark implies that the same is true for the embeddings $i$ and $i^{\prime}$.

The correspondence $X \mapsto X[\Phi]$ is functorial in the following sense. Let $f: X \rightarrow Y$ be a morphism of fibrewise pointed spaces $A \stackrel{s}{\longrightarrow} X \stackrel{p}{\longrightarrow} A$ and

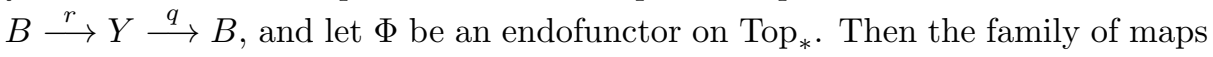

$$
\Phi\left(\left.f\right|_{X_{a}}\right): \Phi\left(X_{a}, s(a)\right) \rightarrow \Phi\left(Y_{q f s(a)}, f s(a)\right), \quad a \in A
$$

determines the function

$$
f[\Phi]:=\coprod_{a \in A} \Phi\left(\left.f\right|_{X_{a}}\right): \coprod_{a \in A} \Phi\left(X_{a}, s(a)\right) \longrightarrow \coprod_{b \in B} \Phi\left(Y_{b}, r(b)\right) .
$$

To prove the continuity $f[\Phi]$ we need some assumptions.

TheOREM 2.4. Assume that there are closed embeddings $i: X \rightarrow E$ and $i^{\prime}: Y \rightarrow E^{\prime}$ into countable products of real lines, and that $\Phi$ is a topologically continuous functor that preserves closed real subspaces. Then $f[\Phi]: X[\Phi] \rightarrow$ $Y[\Phi]$ is continuous.

Proof. By Tietze extension theorem there is a map $u$ that makes commutative the following diagram:

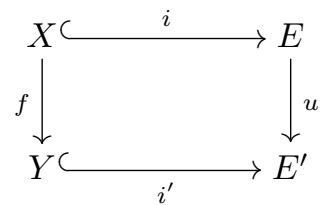


Then the following diagram of embeddings

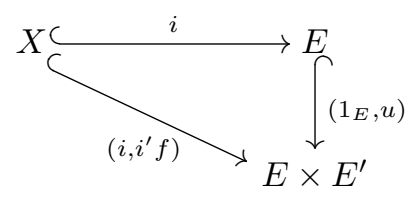

together with Theorem 2.3 imply that the topology of $X[\Phi]$ is determined by the embedding $\left(i, i^{\prime} f\right): X \rightarrow E \times E^{\prime}$.

For every $a \in A$ we have the commutative diagram

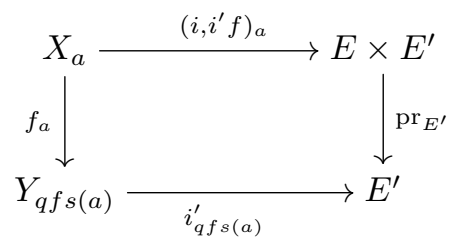

because for $x \in X_{a}$

$$
\begin{aligned}
\operatorname{pr}_{E^{\prime}}\left(i, i^{\prime} f\right)_{a}(x) & =\operatorname{pr}_{E^{\prime}}\left(i(x)-i(s(a)), i^{\prime} f(x)-i^{\prime} f s(a)\right. \\
& =i^{\prime} f(x)-i^{\prime} f s(a)=i_{q f s(a)}^{\prime}(f(x)) .
\end{aligned}
$$

Thus we obtain the commutative diagram

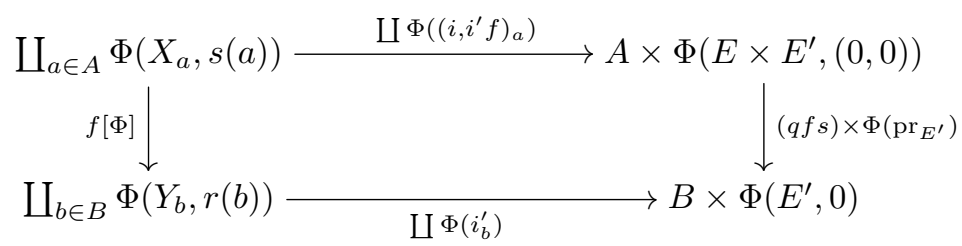

which by the universal property of initial topologies implies that $f[\Phi]$ is continuous.

It is much easier to show that a natural transformation $\mu$ : $\Phi \rightarrow \Psi$ induces a map from $X[\Phi]$ to $X[\Psi]$.

Proposition 2.5. Given a fibrewise pointed space $B \stackrel{s}{\longrightarrow} X \stackrel{p}{\longrightarrow} B$ with $X$ a Tikhonov space and endofunctors $\Phi, \Psi$ of $\mathrm{Top}_{*}$, then every natural transformation $\mu: \Phi \rightarrow \Psi$ induces a fibrewise pointed map $X[\mu]: X[\Phi] \rightarrow X[\Psi]$.

Proof. The continuity of $X[\mu]$ follows from the universal property of initial topologies and the commutativity of the following diagram

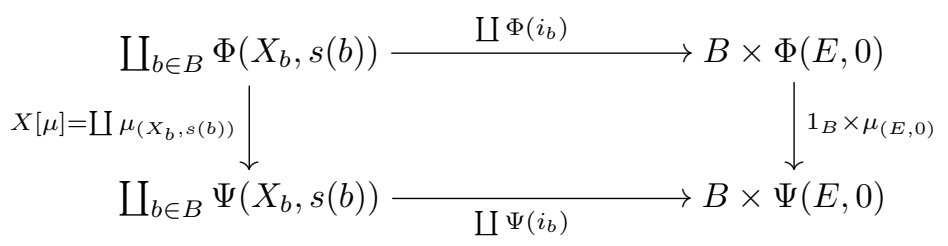


Given a fibrewise pointed space If $B \stackrel{s}{\longrightarrow} X \stackrel{p}{\longrightarrow} B$ and a map $f: A \rightarrow B$ let $A \sqcap X$ denote the pullback of $X$ along $f$, i.e. $A \sqcap X=\{(a, x) \in A \times X \mid f(a)=$ $p(x)\} . A \sqcap X$ is a fibrewise pointed space over $A$ with respect to the section $\bar{s}: A \rightarrow A \sqcap X$ given by $\bar{s}(a):=(a, s f(a))$. The projection $\operatorname{pr}_{X}: A \sqcap X \rightarrow X$ is a morphism of fibrewise pointed spaces, so if $X$ and $\Phi$ satisfy the assumptions of Theorem 2.4, we obtain a map of fibrewise pointed spaces $\operatorname{pr}_{X}[\Phi]:(A \sqcap X)[\Phi] \rightarrow$ $X[\Phi]$. By the universal property of pullbacks, $\operatorname{pr}_{X}[\Phi]$ factors through a map $(A \sqcap X)[\Phi] \rightarrow A \sqcap X[\Phi]$, which is clearly a homeomorphism.

Proposition 2.6. Let $X$ be a fibrewise pointed space over $B$ and $f: A \rightarrow B$ be any map. If $X$ and $\Phi$ satisfy the assumptions of Theorem 2.4 then there is a natural isomorphism of fibrewise pointed spaces $(A \sqcap X)[\Phi] \cong A \sqcap X[\Phi]$ over $A$.

If we assume that $\Phi$ preserves real subspaces and extend its action to the trivial fibrewise pointed space

$$
B \stackrel{1 \times c_{x_{0}}}{\longrightarrow} B \times\left(F, x_{0}\right) \stackrel{\text { pr }}{\longrightarrow} B
$$

then the maps $i_{b}$ used in the definition are all equal, so that $\left(B \times\left(F, x_{0}\right)\right)[\Phi]$ and $B \times \Phi\left(F, x_{0}\right)$ coincide as fibrewise pointed spaces over $B$. Thus we get the following result.

Proposition 2.7. If $\Phi: \mathrm{Top}_{*} \rightarrow \mathrm{Top}_{*}$ preserves real subspaces and if $X$ is (locally) trivial as a fibrewise pointed space over $B$ then $X[\Phi]$ is also (locally) trivial as a fibrewise pointed space over $B$.

We conclude the section by considering thet case when $B \stackrel{s}{\longrightarrow} X \stackrel{p}{\longrightarrow} B$ is a fibrewise pointed fibration in the sense of [11, Definition 8.1.1]. This is to say that the projection $p: X \rightarrow B$ is a (Hurewicz) fibration and the corresponding lifting function $\Gamma: B^{I} \sqcap X \rightarrow X^{I}$ (cf. [12, Theorem 1.1]) may be chosen so that $\Gamma(\alpha, s \alpha(0))(t)=s \alpha(t)$ for every $\alpha: I \rightarrow B$. In other words, for every $\alpha: I \rightarrow B$ and every $t \in I$ the map

$$
f_{\alpha, t}: X_{\alpha(0)} \rightarrow X_{\alpha(t)}, \quad f_{\alpha, t}(x):=\Gamma(\alpha, x)(t)
$$

is base-point preserving.

THEOREM 2.8. Let $B \stackrel{s}{\longrightarrow} X \stackrel{p}{\longrightarrow} B$ be a fibrewise pointed fibration and let $\Phi$ be a topologically continuous endofunctor of $\mathrm{Top}_{*}$. If $X$ admits a closed embedding into a countable product of real lines and if $\Phi$ preserves closed real subspaces, then $B \stackrel{s_{\Phi}}{\longrightarrow} X[\Phi] \stackrel{p_{\Phi}}{\longrightarrow} B$ is also a fibrewise pointed fibration.

Proof. Let us define $\Gamma_{\Phi}: B^{I} \sqcap X[\Phi] \rightarrow X[\Phi]^{I}$ as $\Gamma_{\Phi}(\alpha, u)(t):=\Phi\left(f_{\alpha, t}\right)(u)$. It is easy to verify that $\Gamma_{\Phi}$ is a lifting function in a fibrewise pointed sense, 
so it only remains to prove its continuity. In fact, it is sufficient to prove the continuity of the adjoint map $\widehat{\Gamma}_{\Phi}:\left(B^{I} \times I\right) \sqcap X[\Phi] \rightarrow X[\Phi]$, given by

$$
\widehat{\Gamma}_{\Phi}(\alpha, t, u):=\Phi\left(f_{\alpha, t}\right)(u) .
$$

The commutativity of the following diagram

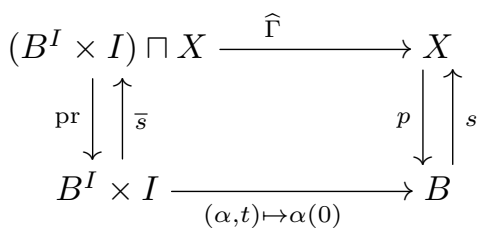

(where $\bar{s}(\alpha, t)=(\alpha, t, s \alpha(0)))$ shows that $\widehat{\Gamma}$ is a morphism of fibrewise pointed spaces. Clearly, $\widehat{\Gamma}_{\Phi}=\widehat{\Gamma}[\Phi]$, which by Theorem 2.4 implies that $\widehat{\Gamma}_{\Phi}$ is continuous.

\section{Fibrewise pointed LS category}

In this section we use the extension of functors to perform a straightforward construction of the Whitehead-Ganea diagram for the fibrewise LusternikSchnirelmann category. Let us first recall the relevant facts about the classical theory (see [1] for more details and historical notes). A subset $A$ is categorical in $X$ if it can be contracted in $X$ to a point (in other words, if the inclusion $A \hookrightarrow X$ is nul-homotopic). The Lusternik-Schnirelmann (LS) category of $X$, denoted $\operatorname{cat}(X)$, is the minimal $n$ such that $X$ can be covered by $n$ open categorical subsets. The LS category is a homotopy invariant of $X$ but the above definition is not practical for homotopy theoretical considerations. G.W. Whitehead proved that under mild assumptions on $X$ (normal, path-connected and with a non-degenerate base-point $\left.x_{0} \in X\right)$ one can determine cat $(X)$ by considering only subsets $A \subseteq X$ that are strongly categorical in the sense that there is a base-point preserving deformation $H: X \times I \rightarrow X$ such that $H_{0}=1_{X}$ and $H_{1}(A)=x_{0}$. Then one immediately obtains the following characterization of $\operatorname{cat}(X)$ : let $W^{n}\left(X, x_{0}\right)$ be the $n$-th 'fat wedge' of $X$,

$$
W^{n}\left(X, x_{0}\right):=\left\{\left(x_{1}, \ldots, x_{n}\right) \in X^{n} \mid x_{i}=x_{0} \text { for some } i\right\}
$$

(observe that the quotient $X^{n} / W^{n}\left(X, x_{0}\right)$ is precisely the $n$-fold smash product $\left.\wedge^{n}\left(X, x_{0}\right)\right)$; then $\operatorname{cat}(X)$ is the minimal $n$ such that there is a homotopy lifting of the $n$-fold diagonal $\Delta_{n}: X \rightarrow X^{n}$ to $W^{n}\left(X, x_{0}\right)$ as in the diagram

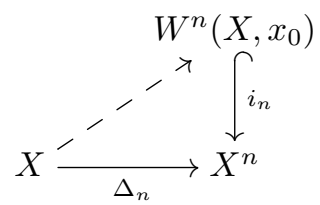


There is also a companion characterization of the LS category introduced by T. Ganea: he associates to $X$ a series of fibrations $p_{n}: G_{n}\left(X, x_{0}\right) \rightarrow X$ where $G_{1}\left(X, x_{0}\right)$ is the space of based paths from $(I, 0)$ to $\left(X, x_{0}\right)$ with $p_{1}$ the usual path-fibration, and $p_{n+1}$ is obtained from $p_{n}$ by turning the induced map $G_{n}\left(X, x_{0}\right) / p^{-1}\left(x_{0}\right) \rightarrow X$ into a fibration. Then the following is the $(n$-th) Whitehead-Ganea diagram associated to $X$ :

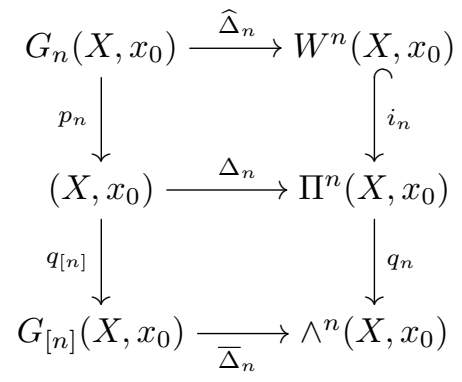

In this diagram we find all functors and natural transformations that will be used later: the $n$-fold product $\Pi^{n}$, the $n$-fold fat wedge $W^{n}$, the $n$-fold smash product $\wedge^{n}$, the $n$-th Ganea space functor $G_{n}$, and the $n$-th Ganea cofibre functor $G_{[n]}$ (the cofibre of the projection $p_{n}$ ). The maps $q_{n}$ and $q_{[n]}$ are respectively the cofibre maps of $i_{n}$ and $p_{n}$, while $\widehat{\Delta}_{n}$ and $\bar{\Delta}_{n}$ are induced by the $n$-fold diagonal $\Delta_{n}$. Observe that all of the above are endofunctors on Top ${ }_{*}$, so we keep track of the base-points in the notation as that will be of crucial importance later on. Moreover, all maps that appear in the diagram are in fact natural transformations. It also turns out that the upper square is a homotopy pullback (see [1, Theorem 1.63]), which immediately implies that cat $(X)$ is precisely the minimal $n$ for which the $n$-th Ganea fibration admits a section. From the Whitehead-Ganea we may derive a series of estimates and approximations of $\operatorname{cat}(X)$ (cf. [1, Chapter 2]) so it is justly considered to be a cornerstone of the homotopy theoretic approach to LS category.

The fibrewise LS category was introduced by I. James and J. Morris [10]: given a fibrewise pointed space $B \stackrel{s}{\longrightarrow} X \stackrel{p}{\longrightarrow} B$, a subset $A \subseteq X$ is (fibrewise pointed) categorical if the inclusion $A \subseteq X$ is nul-homotopic in the sense of fibrewise pointed spaces, i.e. if there is a homotopy $H: A \times I \rightarrow X$ that vertically deforms $A$ to a subset of $s(B)$. The minimal number of fibrewise pointed categorical subsets that cover $X$ is the fibrewise pointed category of $X$, denoted cat $_{B}^{*}(X)$. James and Morris proved (see [10, Propositions 6.1 and 6.2]) that when $X$ is path-connected, fully normal and with a non-degenerate section, then its fibrewise pointed category may be computed by considering only strongly categorical subsets, i.e. such that $s(B) \subseteq A$ and that there is a vertical deformation of $X$ that fixes $s(B)$ and contracts $A$ to $s(B)$. Thus they obtain a Whiteheadtype characterization of the fibrewise pointed category of $X$ as the minimal $n$ 
for which the fibrewise pointed diagonal into the $n$-fold fibrewise pointed product of $X$ can be vertically deformed into the $n$-fold fibrewise pointed fat wedge of $X$. In [10] both the fibrewise pointed product and the fibrewise pointed fat wedge are constructed directly. In fact, one may proceed in a similar vein to construct directly the fibrewise pointed Ganea space and other spaces (and morphisms) that fit in a fibrewise pointed version of the Whitehead-Ganea diagram for a given fibrewise pointed space.

The otherwise standard procedure that we have sketched seems to be unnecessarily long and repetitive, so it would be preferable to have a more efficient and conceptual approach. The tool of choice is the extension of functors that we developed in the previous section. As a base we take the endofunctors of Top and their natural transformations that appear in the classical Whitehead-Ganea diagram, namely: the functors Id, $\Pi^{n}, W^{n}, \wedge^{n}, G_{n}$ and $G_{[n]}$ and their natural transformations that are summarized in the following diagram

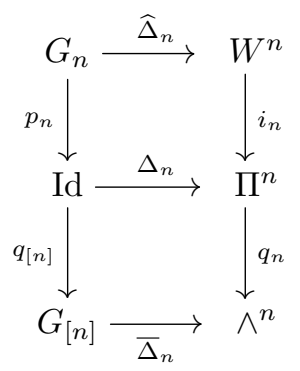

Given a fibrewise pointed Tikhonov space $X$ over $B$ we simply apply the extension of functors procedure together with Proposition 2.5 to obtain a diagram of fibrewise pointed spaces that is the Whitehead-Ganea diagram for the fibrewise pointed LS category of $X$ :

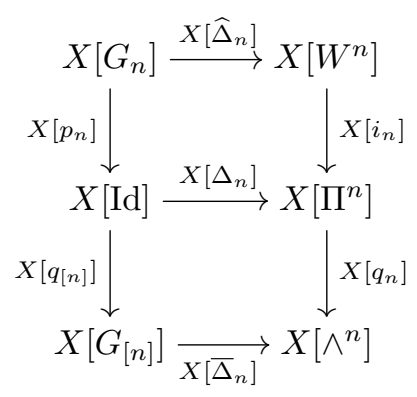

It is now easy to show (directly or by invoking the results of James and Morris) that $\operatorname{cat}_{B}^{*}(X)$ is the minimal integer $n$ for which $X\left[\Delta_{n}\right]$ admits a homotopy lifting along $X\left[i_{n}\right]$ or equivalently, the minimal integer for which $X\left[p_{n}\right]$ admits a section. Furthermore, one may exploit the relations between the various spaces and maps in the above diagram to develop various estimates for the topological 
complexity as in [4] and [5], see also the discussion on the weak fibrewise category in $[2$, Section 19].

\section{REFERENCES}

[1] O. Cornea, G. Lupton, J. Oprea and D. Tanré, Lusternik-Schnirelmann Category, AMS, Mathematical Surveys and Monographs, vol. 103, 2003.

[2] M.C. Crabb And I.M. James, Fibrewise Homotopy Theory, Springer Verlag, 1998.

[3] A. Dold, A simple proof of the Jordan-Alexander complement theorem, Amer. Math. Monthly 100 (1993), 856-858.

[4] A. Franc and P. PAVEšić, Lower bounds for topological complexity, Topology Appl. 160 (2013), 991-1004.

[5] — Spaces with high topological complexity, Proc. Roy. Soc. Edinburgh (to appear).

[6] D. Husemoller, Fibre Bundles, 3rd ed., Graduate Texts in Mathematics, Vol. 20, Springer Verlag, 1994.

[7] I. IwaSE AND M. SAKaI, Functors on the category of quasi-fibrations, Topology Appl. 155 (2008), 1403-1409.

[8] - Topological complexity is a fibrewise L-S category. Topology Appl. 157 (2010), no. $1,10-21$.

[9] I.M. James, General Topology and Homotopy Theory, Springer Verlag, 1984.

[10] I.M. James And J.R. Morris, Fibrewise category, Proc. Roy. Soc. Edinburgh 119A (1991), 177-190.

[11] J.P. May and J. Sigurdsson, Parametrized Homotopy Theory, Mathematical Surveys and Monographs 132, AMS Providence, RI, 2006.

[12] P. Pavešić And R.A. Piccinini, Fibrations and their Classification, Research and Exposition in Mathematics, Vol. 33, Heldermann Verlag, 2013.

[13] A.H. Stone, Paracompactness and product spaces, Bull. Amer. Math. Soc. 54 (1948), 977-982.

Petar PavešIć

Faculty of Mathematics and Physics

University of Ljubljana

Jadranska 21

1000 Ljubljana, SLOVENIA

E-mail address: petar.pavesic@fmf.uni-lj.si 